\title{
Analytical calculation of Stokes profiles of rotating stellar magnetic dipole
}

\author{
M. J. Martínez González \& A. Asensio Ramos \\ Instituto de Astrofísica de Canarias, Vía Láctea s/n, 38200, La Laguna, Tenerife, Spain \\ Departamento de Astrofísica, Universidad de La Laguna, E-38205 La Laguna, Tenerife, \\ Spain
}

Received —

Submitted to the Astrophysical Journal 


\begin{abstract}
The observation of the polarization emerging from a rotating star at different phases opens up the possibility to map the magnetic field in the stellar surface thanks to the well-known Zeeman Doppler Imaging. When the magnetic field is sufficiently weak, the circular and linear polarization profiles locally in each point of the star are proportional to the first and second derivatives of the unperturbed intensity profile, respectively. We show that the weak-field approximation (for weak lines in the case of linear polarization) can be generalized to the case of a rotating star including the Doppler effect and taking into account the integration on the stellar surface. The Stokes profiles are written as a linear combination of wavelength-dependent terms expressed as series expansions in terms of Hermite polynomials. These terms contain the surface integrated magnetic field and velocity components. The direct numerical evaluation of these quantities is limited to rotation velocities not larger than 8 times the Doppler width of the local absorption profiles. Additionally, we demonstrate that, in a rotating star, the circular polarization flux depends on the derivative of the intensity flux with respect to the wavelength and also on the profile itself. Likewise, the linear polarization depends on the profile and on its first and second derivative with respect to the wavelength. We particularize the general expressions to a rotating dipole.
\end{abstract}

Subject headings: Stars: magnetic fields, rotation — Techniques: polarimetric — Methods: analytical 


\section{Introduction}

The present knowledge of solar and stellar magnetism derives essentially from the interpretation of polarization in spectral lines due to the Zeeman effect 1 . In the presence of a weak magnetic field, the radiative transfer equation for polarized light can be solved analytically and it is known as the "weak field approximation". Although very simple, it relies on a set of strong assumptions that we discuss later. However, the weak field approximation is applicable in many scenarios and gives very good results for the inference of magnetic fields as compared with more elaborate methods. In solar physics, it is at the heart of the success of a large number of synoptic magnetographs, like those of Big Bear (Varsik 1995; Spirock et al. 2001). It has been also used recently to produce vector magnetograms with the Imaging Magnetograph Experiment (IMaX) instrument (Martínez Pillet et al. 2011) onboard the Sunrise balloon (Solanki et al. 2010). In stellar spectropolarimetry, the weak field approximation is at the base of the least-squares deconvolution (LSD; Donati et al. 1997), the most successful technique used to detect and measure magnetic fields in solar-type stars, or in some recent works on central stars of planetary nebulae (Jordan et al. 2005; Leone et al. 2011), white dwarfs (Aznar Cuadrado et al. 2004), pulsating stars (Silvester et al. 2009), hot subdwarfs (O'Toole et al. 2005) and Ap and Bp stars (Wade et al. 2000; Bagnulo et al. 2002).

Most of the scientific cases commented so far deal with unresolved structures which means that the weak field approximation has to be understood in terms of fluxes of the Stokes parameters instead of specific intensities. Recently, Martínez González et al. (2012) derived the weak field expressions in terms of fluxes, particularizing to the case of the

\footnotetext{
${ }^{1}$ There are some notable exceptions as, for example, the study of the quiet Sun magnetism by means of the interpretation of scattering polarization and its modification by the Hanle effect (e.g., Trujillo Bueno 2011, for a recent review)
} 
stellar dipole. They neglected rotation to simplify the equations and to derive analytical expressions for the inference of the magnetic field. When rotation is taken into account (in other words, there is a correlation between the magnetic field and the line-of-sight velocity), the problem becomes much more challenging and it is usually solved numerically (e.g., Petit \& Wade 2012, for a recent effort). However, we show in this paper that it is possible to obtain analytical expressions for the Stokes vector fluxes in the weak field approximation. There are two interests on this effort. First, having an analytical expression for the Stokes flux might be of help to investigate the interplay between rotation and the magnetic field. Second, it can be used to speed up inversion codes based on the weak-field approximation because no numerical integration is needed. This will facilitate the application of Bayesian

inference codes, like the one developed by Petit \& Wade (2012), that are based on Markov Chain Monte Carlo methods that need to carry out the synthesis thousands of times to sample the posterior distribution.

This is the first paper of a series dealing with analytical expressions for the fluxes of the Stokes parameters in the weak field limit. This paper deals with the expressions for a rotating star with a magnetic field on its surface. The results depend on the correlation between the magnetic field and the velocity and are general for any rotation profile or any magnetic field configuration. We particularize the equations to the case of a rotating magnetic stellar dipole.

\section{The weak field approximation for a star}

Let us consider a star with arbitrary velocity and magnetic fields on its surface. We define both fields for an arbitrary point $\mathbf{r}$ in the surface using the reference frame represented in Fig. 1. The line-of-sight (LOS) is along the $Z$ axis, while we choose the axis $X$ as the one defining the reference direction for positive Stokes $Q$. 


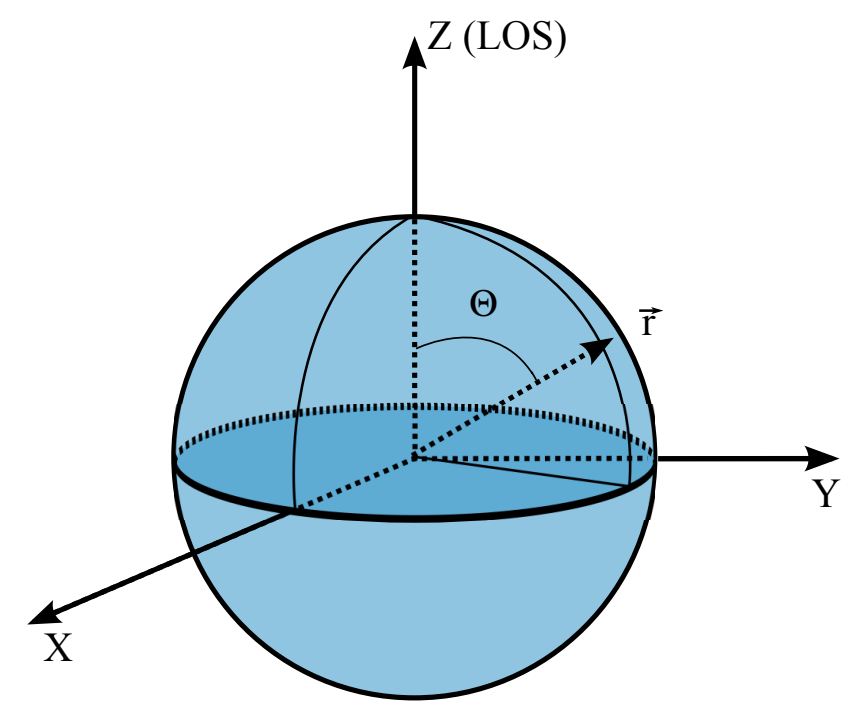

Fig. 1. - This figure shows the reference systems used to define the Stokes parameters in this work. We consider that the observer is along the $Z$ axis. The direction $X$ defines the reference for $Q>0$. The vector $\mathbf{r}$ defines any position on the stellar surface.

Our aim is to compute the local Stokes profiles emerging from every point of the visible surface and compute its integral. As explained in the introduction, a simple (although subject to approximations) way to relate the emergent Stokes profiles with the magnetic field vector is the weak-field approximation. This approximation (combined with the weak-line approximation for linear polarization) leads to an analytical solution to the radiative transfer equation. It is based on the following three fundamental assumptions: i) the magnetic field vector is constant along the line of sight on the region of the stellar atmosphere where the spectral line is formed, ii) the magnetic field intensity is sufficiently weak so that the Zeeman splitting is smaller than any other line broadening mechanism and iii) the LOS velocity and any broadening mechanism are constant with height in the line formation region. Under these simplifying conditions, the magnetic field can be considered as a perturbation to the zero-field case. This approximation is not strictly valid in general, but it is a good approximation whenever the magnetic field 
strength is weak in the line formation region in the absence of strong gradients along the LOS. Quantitatively, the approximation holds whenever $g \Delta \lambda_{B} \ll \Delta \lambda_{D}$, where $\Delta \lambda_{B}$ (see Eq. (3.14) in Landi Degl'Innocenti \& Landolfi 2004) is the Zeeman splitting that is proportional to the field strength, $\Delta \lambda_{D}$ is the dominant broadening mechanism and $g$ represents the effective Landé factor of the line, which quantifies the magnetic sensitivity of the line and that only depends on the quantum numbers of the transition (see, e.g., Landi Degl'Innocenti \& Landolfi 2004). From its definition, it is clear that the weak field regime occurs at different field strengths for different spectral lines, for different stellar objects and for different spectral resolutions. For instance, for a magnetically sensitive iron spectral line with $g=3$ at $5000 \AA$ with a standard thermal broadening at $T=6500 \mathrm{~K}$, the weak-field regime is applicable for fields below $\sim 600 \mathrm{G}$ if the spectral resolution of the spectrograph is smaller than the line broadening.

Under the previous simplifying assumptions, and to first order in $\Delta \lambda_{B}$, the intensity profile $I(\lambda)$ of a spectral line formed in a weak magnetic field is fully insensitive to the magnetic field. In other words, it fulfills the standard radiative transfer equation in the absence of a magnetic field (e.g., Mihalas 1978). Likewise, at first order in $\Delta \lambda_{B}$, the circular polarization profile, i.e., the Stokes $V(\lambda)$ profile for a given spectral line, has the following expression:

$$
V(\lambda)=-C \lambda_{0}^{2} g B_{z} \frac{\partial I(\lambda)}{\partial \lambda}
$$

where the symbol $B_{z}$ stands for the longitudinal component of the magnetic field (the projection along the LOS in the reference system shown in Fig. 1). The symbol $\lambda$ stands for the wavelength while $\lambda_{0}$ is the central wavelength of the transition. The constant $C=4.67 \times 10^{-13} \mathrm{G}^{-1} \AA^{-1}$.

At first order in $\Delta \lambda_{B}$, linear polarization is zero. In order to obtain an expression for the Stokes profiles characterizing linear polarization we have to expand the radiative 
transfer equation to second order in $\Delta \lambda_{B}$ and assume that the spectral line is not saturated and weak. Under these assumptions, a good approximation to the intensity profile emerging from the stellar atmosphere is to consider it, in general, proportional to a Voigt function (see Eqs. (9.84) and (9.86) in Landi Degl'Innocenti \& Landolfi 2004). For the sake of simplicity, we consider the case of a Gaussian line profile. All our results can be derived for a Voigt profile but the final expressions are more complicated without any fundamental difference. This assumption somehow reduces the scope of applicability of our results for linear polarization because the line shape self-similarity is only valid for lines whose line depth is smaller than $\sim 40 \%$ according to the results of Kochukhov et al. (2010).

With this assumption, the weak-field approximation for the Stokes $Q(\lambda)$ and $U(\lambda)$, defined in the $X Y Z$ reference system shown in Fig. 1, are (see Landi Degl'Innocenti \& Landolfi 2004; Martínez González et al. 2012):

$$
\begin{aligned}
& Q(\lambda)=-\frac{C^{2}}{4} \lambda_{0}^{4} G\left(B_{x}^{2}-B_{y}^{2}\right) \frac{\partial^{2} I(\lambda)}{\partial \lambda^{2}} \\
& U(\lambda)=-\frac{C^{2}}{2} \lambda_{0}^{4} G B_{x} B_{y} \frac{\partial^{2} I(\lambda)}{\partial \lambda^{2}}
\end{aligned}
$$

where the symbol $G$ plays the role of the effective Landé factor for linear polarization and quantifies the sensitivity of linear polarization to the magnetic field. Again, it is only a function of the quantum numbers of the transition (Landi Degl'Innocenti \& Landolfi 2004). The symbols $B_{x}$ and $B_{y}$ stand for the components of the magnetic field vector along the $X$ and $Y$ axes, respectively. The Stokes profiles in any other reference system that is defined as a rotation around the $Z$ axis by an angle $\gamma$ are given by the usual rotation (Landi Degl'Innocenti \& Landolfi 2004):

$$
\begin{aligned}
Q_{\text {new }}(\lambda) & =\cos (2 \gamma) Q(\lambda)+\sin (2 \gamma) U(\lambda) \\
U_{\text {new }}(\lambda) & =-\sin (2 \gamma) Q(\lambda)+\cos (2 \gamma) U(\lambda),
\end{aligned}
$$

where the quantities with the subindex "new" refer in this case to the new reference frame. 
When observing unresolved objects, the detected polarized radiation is obtained as an integration in the plane of the sky of the individual Stokes parameters at each point of the stellar surface $(\Sigma)$, all of them referred to a common reference system. We describe the stellar disk using the polar coordinates $\rho$ and $\theta$, where $\rho$ is normalized to the stellar radius, so that its range is from 0 to 1 (see Martínez González et al. 2012). Therefore, the average of any arbitrary function $h(\rho, \theta)$ on the surface $\Sigma$ will be given by:

$$
\bar{h}=\iint d \Sigma h(\rho, \theta)=\int_{0}^{1} \rho d \rho \int_{0}^{2 \pi} d \theta h(\rho, \theta) .
$$

As a consequence, it is obvious that the observed values of the Stokes flux vector depends on the surface distribution of the magnetic field, on the centre-to-limb variation (CLV) of the radiation, and on the Doppler effect due to the rotation of the star.

Following standard approximations and assuming that the star has azimuthal symmetry, Martínez González et al. (2012) parameterized the intensity at each point of the star as a product of two functions: $I_{0}(\lambda)$, that only depends on the wavelength (the intensity at the disc centre since they neglect rotation), and $f(\mu)$, the CLV that depends on the position on the disk and is wavelength independent (e.g., Claret 2000; Cox 2000):

$$
f(\mu)=\left(1-a-b+a \mu+b \mu^{2}\right)
$$

The CLV is given in terms of $\mu=\cos \Theta=\sqrt{1-\rho^{2}}$, where $\Theta$ is the astrocentric angle between the normal to a point in the stellar surface and the line of sight (see Fig. 1). The parameters $a$ and $b$ have values between 0 and 1 and are supposed to be constant along the spectral line (although they can vary from line to line). With this assumption, we also force the line depth to be $\mu$-independent. Note that the values of $a$ and $b$ have to fulfill the condition that the intensity has to be positive. This simple parameterization allowed them to express the weak field equations in terms of the observed fluxes, thus mimicking the expressions for the specific Stokes vector. This was possible because the intensity flux 
is assumed to be the product of the wavelength-dependent intensity at disk center and the wavelength independent CLV. Using this relationship, the weak-field and weak-line approximation for the integrated polarized flux remains formally the same than for the specific Stokes vector but the components of the magnetic field appear weighted by the CLV law.

When the velocity field is included, the situation turns out to be much more complicated because the intensity itself depends on the local projection of the velocity along the $Z$ axis $\left(v_{z}\right)$, which subsequently depends on the position along the stellar disk in a potentially complicated way. In this case, the fluxes of the Stokes vector are expressed as:

$$
\begin{aligned}
& F_{I}(\lambda)=\int_{0}^{1} \rho d \rho \int_{0}^{2 \pi} d \theta I\left(\lambda, v_{z}(\rho, \theta), \mu\right) \\
& F_{Q}(\lambda)=-\frac{C^{2}}{4} \lambda_{0}^{4} G \int_{0}^{1} \rho d \rho \int_{0}^{2 \pi} d \theta\left[B_{x}^{2}(\rho, \theta)-B_{y}^{2}(\rho, \theta)\right] \frac{\partial^{2} I\left(\lambda, v_{z}(\rho, \theta), \mu\right)}{\partial \lambda^{2}} \\
& F_{U}(\lambda)=-\frac{C^{2}}{2} \lambda_{0}^{4} G \int_{0}^{1} \rho d \rho \int_{0}^{2 \pi} d \theta B_{x}(\rho, \theta) B_{y}(\rho, \theta) \frac{\partial^{2} I\left(\lambda, v_{z}(\rho, \theta), \mu\right)}{\partial \lambda^{2}} \\
& F_{V}(\lambda)=-C \lambda_{0}^{2} g \int_{0}^{1} \rho d \rho \int_{0}^{2 \pi} d \theta B_{z}(\rho, \theta) \frac{\partial I\left(\lambda, v_{z}(\rho, \theta), \mu\right)}{\partial \lambda}
\end{aligned}
$$

The immediate consequence of the dependence on $v_{z}(\rho, \theta)$ is that the integrals cannot be separated. To proceed, it is crucial to decouple the rotation velocity and the wavelength to end up with expressions for the weak field approximation in terms of the observed fluxes. In order to do that, we assume that the shape of the local absorption profile is independent of the position on the stellar surface, apart from the Doppler shift introduced by $v_{z}$, and that the CLV is wavelength independent. Furthermore, if we assume that the absorption profile is Gaussian, we end up with:

$I\left(\lambda, v_{z}(\rho, \theta), \mu\right)=f(\mu) I\left(\lambda, v_{z}(\rho, \theta), \mu=1\right)=f(\mu)\left[I_{c}-\eta \exp \left[-\frac{1}{2}\left(\frac{\lambda-\lambda_{0}\left(1+v_{z}(\rho, \theta) / c\right)}{\sigma}\right)^{2}\right]\right]$

where $I_{c}$ stands for the continuum intensity, $\eta$ for the line depth, and $\sigma$ for the line width. 
This approximation is not appropriate for stars with large radial temperature and density gradients because the lines close to the limb will differ strongly from the lines at the disk center. Apart from the Gaussian shape, it is possible to work with Voigt functions but the subsequent expressions turn out to be more complicated and no new physics is gained, so we prefer to stick with the Gaussian shape. The Gaussian shape is also consistent with the assumption of an unsaturated and weak spectral line that is necessary for Eqs. (2) to hold.

We can carry out the Taylor expansion of $I\left(\lambda, v_{z}, \mu=1\right.$ ) around $v_{z}=0$ (we drop now the $\mu$-dependence) to obtain the following expression for the intensity at each point of the stellar surface:

$$
I\left(\lambda, v_{z}\right)=I(\lambda, 0)+\left.\sum_{n=1}^{\infty} \frac{1}{n !} \frac{\partial^{n} I\left(\lambda, v_{z}\right)}{\partial v_{z}}\right|_{v_{z}=0} v_{z}^{n} .
$$

Defining the emission line profile at zero velocity as

$$
\psi(\lambda)=\eta \exp \left[-\frac{1}{2}\left(\frac{\lambda-\lambda_{0}}{\sigma}\right)^{2}\right]
$$

the absorption profile at an arbitrary velocity shift can be written as:

$$
I\left(\lambda, v_{z}\right)=I_{c}-\psi(\lambda)+\left.\sum_{n=1}^{\infty} \frac{1}{n !} \frac{\partial^{n} I\left(\lambda, v_{z}\right)}{\partial v_{z}}\right|_{v_{z}=0} v_{z}^{n}
$$

Taking into account that the Gaussian function is the generating function of the Hermite polynomials:

$$
\frac{d^{n}}{d x^{n}} \exp \left[-\frac{x^{2}}{2}\right]=(-1)^{n} \exp \left[-\frac{x^{2}}{2}\right] H e_{n}(x),
$$

where $H e_{n}(x)$ is the Hermite polynomial of order $n$ with weight function $w(x)=\exp \left(-x^{2} / 2\right)$, also known as Chebyshev-Hermite polynomials (see Abramowitz \& Stegun 1972). If a Voigt function is used instead for the local profile, the derivatives are more complex and the recurrence relations of Heinzel (1978) can be used. Taking the previous definition into account, the derivatives $(n>0)$ of the intensity profile can be expressed as:

$$
\left.\frac{\partial^{n} I\left(\lambda, v_{z}\right)}{\partial v_{z}^{n}}\right|_{v_{z}=0}=-\left(\frac{\lambda_{0}}{\sigma c}\right)^{n} \psi(\lambda) H e_{n}\left(\frac{\lambda-\lambda_{0}}{\sigma}\right)
$$


so that the intensity profile of Eq. (7) is given by the following Gram-Charlier series:

$$
I\left(\lambda, v_{z}, \mu\right)=f(\mu)\left[I_{c}-\psi(\lambda) \sum_{n=0}^{\infty} \frac{1}{n !}\left(\frac{\lambda_{0}}{\sigma c}\right)^{n} H e_{n}\left(\frac{\lambda-\lambda_{0}}{\sigma}\right) v_{z}^{n}\right]
$$

where we have extended the summation to $n=0$ to include the profile at zero velocity, since $H e_{0}(x)=1$. The previous formula shows that the intensity is given as the addition of infinite terms (potentially a large number of them for obtaining convergence) given by the product of a function that depends on the wavelength and another one that depends on the velocity along the line of sight. The latter is the only one that changes on the stellar surface, so that this variable separation allows us to carry out the surface integration. In order to simplify the following computations, it is interesting to define the function

$$
\mathcal{H}\left(\lambda, v_{z}\right)=\sum_{n=0}^{\infty} \frac{1}{n !}\left(\frac{\lambda_{0}}{\sigma c}\right)^{n} H e_{n}\left(\frac{\lambda-\lambda_{0}}{\sigma}\right) v_{z}^{n}
$$

so that the intensity profile and its first and second derivatives with respect to the wavelength are given by:

$$
\begin{aligned}
I\left(\lambda, v_{z}, \mu\right) & =f(\mu)\left[I_{c}-\psi(\lambda) \mathcal{H}\left(\lambda, v_{z}\right)\right] \\
I^{\prime}\left(\lambda, v_{z}, \mu\right) & =-\psi^{\prime}(\lambda) f(\mu) \mathcal{H}\left(\lambda, v_{z}\right)-\psi(\lambda) f(\mu) \mathcal{H}^{\prime}\left(\lambda, v_{z}\right) \\
I^{\prime \prime}\left(\lambda, v_{z}, \mu\right) & =-\psi^{\prime \prime}(\lambda) f(\mu) \mathcal{H}\left(\lambda, v_{z}\right)-2 \psi^{\prime}(\lambda) f(\mu) \mathcal{H}^{\prime}\left(\lambda, v_{z}\right)-\psi(\lambda) f(\mu) \mathcal{H}^{\prime \prime}\left(\lambda, v_{z}\right),
\end{aligned}
$$

where the' and "symbols represent the first and second derivatives with respect to the wavelength.

Applying the previous developments and dropping the dependences of the variables to avoid crowding, the polarized flux in the weak-field weak-line approximation can be written 
as:

$$
\begin{aligned}
F_{I}(\lambda) & =F_{c}-\psi \overline{f \mathcal{H}} \\
F_{Q}(\lambda) & =\frac{C^{2}}{4} \lambda_{0}^{4} G\left[\psi^{\prime \prime}(\lambda) \overline{\left(B_{x}^{2}-B_{y}^{2}\right) f \mathcal{H}}+2 \psi^{\prime}(\lambda) \overline{\left(B_{x}^{2}-B_{y}^{2}\right) f \mathcal{H}^{\prime}}+\psi(\lambda) \overline{\left(B_{x}^{2}-B_{y}^{2}\right) f \mathcal{H}^{\prime \prime}}\right] \\
F_{U}(\lambda) & =\frac{C^{2}}{2} \lambda_{0}^{4} G\left[\psi^{\prime \prime}(\lambda) \overline{B_{x} B_{y} f \mathcal{H}}+2 \psi^{\prime}(\lambda) \overline{B_{x} B_{y} f \mathcal{H}^{\prime}}+\psi(\lambda) \overline{B_{x} B_{y} f \mathcal{H}^{\prime \prime}}\right] \\
F_{V}(\lambda) & =C \lambda_{0}^{2} g\left[\psi^{\prime}(\lambda) \overline{B_{z} f \mathcal{H}}+\psi(\lambda) \overline{B_{z} f \mathcal{H}^{\prime}}\right]
\end{aligned}
$$

where we remind that the overlines represent integration in the plane of the sky, as indicated in Eq. (44). It is important to note that the area asymmetry of the polarized flux is zero as can be verified by computing the wavelength integral of the previous equations (it is easy to verify that the integral is zero by using the properties of the Hermite polynomials).

The symbol $F_{c}$ stands for the continuum intensity flux and it is trivially given by:

$$
F_{c}=\pi I_{c}\left(1-\frac{a}{3}-\frac{b}{3}\right)
$$

As expected, the Stokes parameters depend on quantities averaged over the stellar surface. Using the expansion on Hermite polynomials of the absorption spectral line, the terms appearing in the previous equations can be written as the following summations:

$$
\begin{aligned}
\overline{B_{z} f \mathcal{H}} & =-\frac{1}{2} \sum_{n=0}^{\infty} \frac{1}{n !}\left(\frac{\lambda_{0}}{\sigma c}\right)^{n} H e_{n}\left(\frac{\lambda-\lambda_{0}}{\sigma}\right) X_{1}(n) \\
\overline{\left(B_{x}^{2}-B_{y}^{2}\right) f \mathcal{H}} & =\frac{1}{4} \sum_{n=0}^{\infty} \frac{1}{n !}\left(\frac{\lambda_{0}}{\sigma c}\right)^{n} H e_{n}\left(\frac{\lambda-\lambda_{0}}{\sigma}\right) X_{2}(n) \\
\overline{B_{x} B_{y} f \mathcal{H}} & =\frac{1}{4} \sum_{n=0}^{\infty} \frac{1}{n !}\left(\frac{\lambda_{0}}{\sigma c}\right)^{n} H e_{n}\left(\frac{\lambda-\lambda_{0}}{\sigma}\right) X_{3}(n) \\
\overline{f \mathcal{H}} & =\sum_{n=0}^{\infty} \frac{1}{n !}\left(\frac{\lambda_{0}}{\sigma c}\right)^{n} H e_{n}\left(\frac{\lambda-\lambda_{0}}{\sigma}\right) X_{4}(n) .
\end{aligned}
$$


The functions $X_{i}(n)$ are given by:

$$
\begin{aligned}
& X_{1}(n)=\int_{0}^{1} \rho d \rho \int_{0}^{2 \pi} d \theta f(\mu) B_{z}(\rho, \theta) v_{z}^{n}(\rho, \theta) \\
& X_{2}(n)=\int_{0}^{1} \rho d \rho \int_{0}^{2 \pi} d \theta f(\mu)\left(B_{x}^{2}(\rho, \theta)-B_{y}^{2}(\rho, \theta)\right) v_{z}^{n}(\rho, \theta) \\
& X_{3}(n)=\int_{0}^{1} \rho d \rho \int_{0}^{2 \pi} d \theta f(\mu) B_{x}(\rho, \theta) B_{y}(\rho, \theta) v_{z}^{n}(\rho, \theta) \\
& X_{4}(n)=\int_{0}^{1} \rho d \rho \int_{0}^{2 \pi} d \theta f(\mu) v_{z}^{n}(\rho, \theta) .
\end{aligned}
$$

It is important to note that Eqs. (16) and (18) are formally invariant for any magnetic field configuration, rotation profile and center-to-limb variation once the assumptions stated before (sufficiently weak magnetic field, wavelength independent CLV, unsaturated and weak Gaussian line) are fulfilled. The information about the magnetic configuration, rotation and the CLV is encoded in the $X_{i}(n)$ functions, which may depend on additional external parameters. This is the case of the dipolar field with solid body rotation (see Sec. 4), in which these functions depend on the orientation of the dipole and the angular velocity vector.

The integrated Stokes parameters also depend on terms that include the wavelength derivative of the $\mathcal{H}$ function. Thanks to the effective separation between wavelength and velocity, these are easily obtained from the previous ones by using known properties of the Hermite polynomials. For instance:

$$
\begin{gathered}
\overline{B_{z} f \mathcal{H}^{\prime}}=-\frac{1}{2} \sum_{n=1}^{\infty} \frac{1}{(n-1) !} \frac{1}{\sigma}\left(\frac{\lambda_{0}}{\sigma c}\right)^{n} H e_{n-1}\left(\frac{\lambda-\lambda_{0}}{\sigma}\right) X_{1}(n) \\
\overline{B_{z} f \mathcal{H}^{\prime \prime}}=-\frac{1}{2} \sum_{n=2}^{\infty} \frac{1}{(n-2) !} \frac{1}{\sigma^{2}}\left(\frac{\lambda_{0}}{\sigma c}\right)^{n} H e_{n-2}\left(\frac{\lambda-\lambda_{0}}{\sigma}\right) X_{1}(n),
\end{gathered}
$$

and the rest of terms are computed following the same strategy.

When the star is not rotating, the only terms contributing to the summations in Eq. (18) are those with $n=0$. Likewise, the terms with derivatives of Eqs. (20) are always zero. 


\section{The weak-field approximation for observers}

The polarized fluxes of Eqs. (16) can be used to explain stellar observations using a standard optimization method to fit the functional forms to the observations. However, we note that the expressions for the polarized fluxes depend on $\eta$ and $\sigma$, properties of the local line profiles to which we do not have direct access. It is possible, though, to drop the dependence on $\eta$ from the equations if we use the fact that $\psi(\lambda), \psi^{\prime}(\lambda)$ and $\psi^{\prime \prime}(\lambda)$ can be written in terms of $F_{I}(\lambda), F_{I}^{\prime}(\lambda)$ and $F_{I}^{\prime \prime}(\lambda)$, which are, otherwise, observed quantities. This is the strategy followed by Martínez González et al. (2012) to write the weak-field weak-line approximation for a non-rotating star in terms of the observed fluxes (and the parameters of the CLV and the Gaussian profile). So:

$$
\begin{aligned}
F_{I}^{\prime}(\lambda) & =-\left(\psi \overline{f \mathcal{H}^{\prime}}+\psi^{\prime} \overline{f \mathcal{H}}\right) \\
F_{I}^{\prime \prime}(\lambda) & =-\left(\psi \overline{f \mathcal{H}^{\prime \prime}}+\psi^{\prime \prime} \overline{f \mathcal{H}}+2 \psi^{\prime} \overline{f \mathcal{H}^{\prime}}\right)
\end{aligned}
$$

which gives, after isolation of $\psi(\lambda), \psi^{\prime}(\lambda)$ and $\psi^{\prime \prime}(\lambda)$ :

$$
\begin{aligned}
& F_{Q}(\lambda)=-\frac{C^{2}}{4} \lambda_{0}^{4} G\left[\frac{\overline{\left(B_{x}^{2}-B_{y}^{2}\right) f \mathcal{H}}}{\overline{f \mathcal{H}}} F_{I}^{\prime \prime}+2\left(\frac{\overline{\left(B_{x}^{2}-B_{y}^{2}\right) f \mathcal{H}^{\prime}}}{\overline{f \mathcal{H}}}-\frac{\overline{\left(B_{x}^{2}-B_{y}^{2}\right) f \mathcal{H}} \overline{f \mathcal{H}^{\prime}}}{\overline{f \mathcal{H}}^{2}}\right) F_{I}^{\prime}\right. \\
& +\left(\frac{\overline{\left(B_{x}^{2}-B_{y}^{2}\right) f \mathcal{H}^{\prime \prime}}}{\overline{f \mathcal{H}}}-\frac{\overline{\left(B_{x}^{2}-B_{y}^{2}\right) f \mathcal{H}} \overline{f \mathcal{H}^{\prime \prime}}}{\overline{f \mathcal{H}}^{2}}+2 \frac{\overline{\left(B_{x}^{2}-B_{y}^{2}\right) f \mathcal{H}}{\overline{f \mathcal{H}^{\prime}}}^{2}}{\overline{f \mathcal{H}}^{3}}\right. \\
& \left.\left.-2 \frac{\overline{\left(B_{x}^{2}-B_{y}^{2}\right) \mathcal{H}^{\prime}} \overline{f \mathcal{H}^{\prime}}}{\overline{f \mathcal{H}}^{2}}\right)\left(F_{c}-F_{I}\right)\right] \\
& F_{U}(\lambda)=-\frac{C^{2}}{2} \lambda_{0}^{4} G\left[\frac{\overline{B_{x} B_{y} f \mathcal{H}}}{\overline{f \mathcal{H}}} F_{\mathcal{I}}^{\prime \prime}+2\left(\frac{\overline{B_{x} B_{y} f \mathcal{H}^{\prime}}}{\overline{f \mathcal{H}}}-\frac{\overline{B_{x} B_{y} f \mathcal{H}} \overline{f \mathcal{H}^{\prime}}}{\overline{f \mathcal{H}}^{2}}\right) F_{I}^{\prime}\right. \\
& \left.+\left(\frac{\overline{B_{x} B_{y} f \mathcal{H}^{\prime \prime}}}{\overline{f \mathcal{H}}}-\frac{\overline{B_{x} B_{y} f \mathcal{H}} \overline{f \mathcal{H}^{\prime \prime}}}{\overline{f \mathcal{H}}^{2}}+2 \frac{\overline{B_{x} B_{y} f \mathcal{H}} \overline{f \mathcal{H}^{\prime}}}{\overline{f \mathcal{H}}^{3}}-2 \frac{\overline{B_{x} B_{y} \mathcal{H}^{\prime}} \overline{f \mathcal{H}^{\prime}}}{\overline{f \mathcal{H}}^{2}}\right)\left(F_{c}-F_{I}\right)\right] \\
& F_{V}(\lambda)=-C \lambda_{0}^{2} g\left[\frac{\overline{B_{z} f \mathcal{H}}}{\overline{f \mathcal{H}}} F_{I}^{\prime}+\left(\frac{\overline{B_{z} f \mathcal{H}} \overline{f \mathcal{H}^{\prime}}}{\overline{f \mathcal{H}}^{2}}-\frac{\overline{B_{z} f \mathcal{H}^{\prime}}}{\overline{f \mathcal{H}}}\right)\left(F_{c}-F_{I}\right)\right]
\end{aligned}
$$

These expressions are the generalization of the weak-field weak-line approximation to an unresolved star with an arbitrary velocity and magnetic field on the surface. The dependency 
on the local absorption $\eta$ has disappeared once the observed $F_{I}$ is used. However, the local broadening is still present and has to be either inferred from the observations or fixed to a reasonable value. The set of Eqs. (22) represent a description of the Stokes parameters in terms of observed quantities and also in terms of wavelength-dependent unknowns that are explicit functions of the field and velocity distribution on the star (e.g., $\overline{B_{z} f \mathcal{H}} / \overline{f H}$ ). Both contributions are conveniently separated. In principle, one could use these expressions to obtain information about the magnetism of the star following two strategies. The first one is to parameterize these terms by fixing a model for the magnetic structure and infer the values of the parameters. This is the approach we follow in the next section. The second one is to non-parametrically infer these wavelength-dependent unknowns directly using a suitable inversion procedure. Since this would require additional regularization techniques, a full treatment of the problem would have to be done in a Bayesian framework.

An interesting property of the integrated Stokes parameters when rotation is taken into account is that the circular polarization flux depends on the derivative with wavelength of the flux $\left(F_{I}^{\prime}\right)$ but also on the flux itself $\left(F_{I}\right)$. Likewise, the linear polarization fluxes depend on the flux and its first and second order derivative with respect to the wavelength. When particularizing the polarized flux of Eq. (22) to the non-rotating star, we recover the equations developed by Martínez González et al. (2012). This is a consistency check of our equations.

\section{The rotating magnetic dipole}

Let us consider a star that is rotating with an angular velocity vector $\boldsymbol{\omega}$, as shown in Fig. 2. In this section we use two different reference systems for our computations. The arbitrary reference system $X^{\prime} Y^{\prime} Z$, shown in the left panel of Fig. 2, is the one associated with an observer. The line-of-sight (LOS) is along the $Z$ axis and the axis $X^{\prime}$ (arbitrarily 
chosen by the observer) defines the reference direction for positive Stokes $Q$. The angular velocity vector is defined in this reference system by its modulus $(\omega)$, the inclination angle $(\alpha)$ and the azimuth $(\gamma)$ with respect to the $X^{\prime}$ axis. The second reference system, $X Y Z$, is obtained after a rotation of an angle $\gamma$ around the $Z$ axis, so that the angular velocity vector is now contained in the $X Z$ plane. This second reference system will help us in obtaining the final analytical expressions.

One of the simplest non-trivial configuration of a stellar magnetic field is that of a dipole, whose axis is determined by a unit vector e along the dipole moment and an intrinsic dipolar field. The three components of the unit vector e on the cartesian system $X Y Z$ are:

$$
\begin{aligned}
& e_{x}=\sin i \cos j \\
& e_{y}=\sin i \sin j \\
& e_{z}=\cos i,
\end{aligned}
$$

where $i$ is the inclination of the dipolar moment with respect to the LOS and $j$ is its azimuth with respect to the $X$ axis. In the arbitrary reference system $X^{\prime} Y^{\prime} Z$, the azimuth is transformed to $j^{\prime}=j+\gamma$. The emergent Stokes parameters $Q(\lambda)$ and $U(\lambda)$ are then transformed to the arbitrary reference system following Eq. (31). Note that our notation can be translated to that used by Landolfi et al. (1993) making the transformations (in format ours $\rightarrow$ theirs): $\alpha \rightarrow i, j^{\prime} \rightarrow \Lambda, \gamma \rightarrow \Theta, i \rightarrow l$. Therefore, the cosine of the inclination of the dipole axis with respect to the rotational axis, usually termed $\beta$, is plainly given by the dot product $\boldsymbol{\omega} \cdot \mathbf{e}$, i.e., $\cos \beta=\sin \alpha \sin i \cos j^{\prime}+\cos \alpha \cos i$.

The magnetic field at the surface of the star of a dipole placed at the center of the star 
can be expressed in cartesian coordinates in the $X Y Z$ system as:

$$
\begin{aligned}
& B_{x}=-\frac{B_{p}}{2}\left[e_{x}-3 x\left(x e_{x}+y e_{y}+z e_{z}\right)\right] \\
& B_{y}=-\frac{B_{p}}{2}\left[e_{y}-3 y\left(x e_{x}+y e_{y}+z e_{z}\right)\right] \\
& B_{z}=-\frac{B_{p}}{2}\left[e_{z}-3 z\left(x e_{x}+y e_{y}+z e_{z}\right)\right],
\end{aligned}
$$

where $B_{p}$ is the intensity of the magnetic field at the poles. An arbitrary point $(x, y, z)$ in the visible surface of the star can be written in terms of the polar coordinates on the plane of the sky $\Sigma$ as:

$$
\begin{aligned}
& x=\rho \cos \theta \\
& y=\rho \sin \theta \\
& z=\sqrt{1-\rho^{2}},
\end{aligned}
$$

where we have selected the positive solution for $z$ to isolate the visible half of the star.

For simplicity, we assume that the star is rotating as a solid body because it greatly simplifies the equations (the introduction of a non-rigid rotation is formally identical, except for the computation of the $X_{i}(n)$ integrals), so that:

$$
\mathbf{v}=\boldsymbol{\omega} \times \mathbf{r}
$$

In terms of the polar coordinates on the stellar surface, the component of the velocity along the LOS in the $X Y Z$ reference system is given by:

$$
v_{z}=\omega \rho \sin \alpha \sin \theta
$$

Taking all these definitions into account and after some tedious algebra, it is possible to find relatively simple expressions for the $X_{i}(n)$ integrals, which now depend on the set of parameters $\{i, j, \alpha, \omega, n, a, b\}$. The procedure is to plug the functional form of the dipolar 

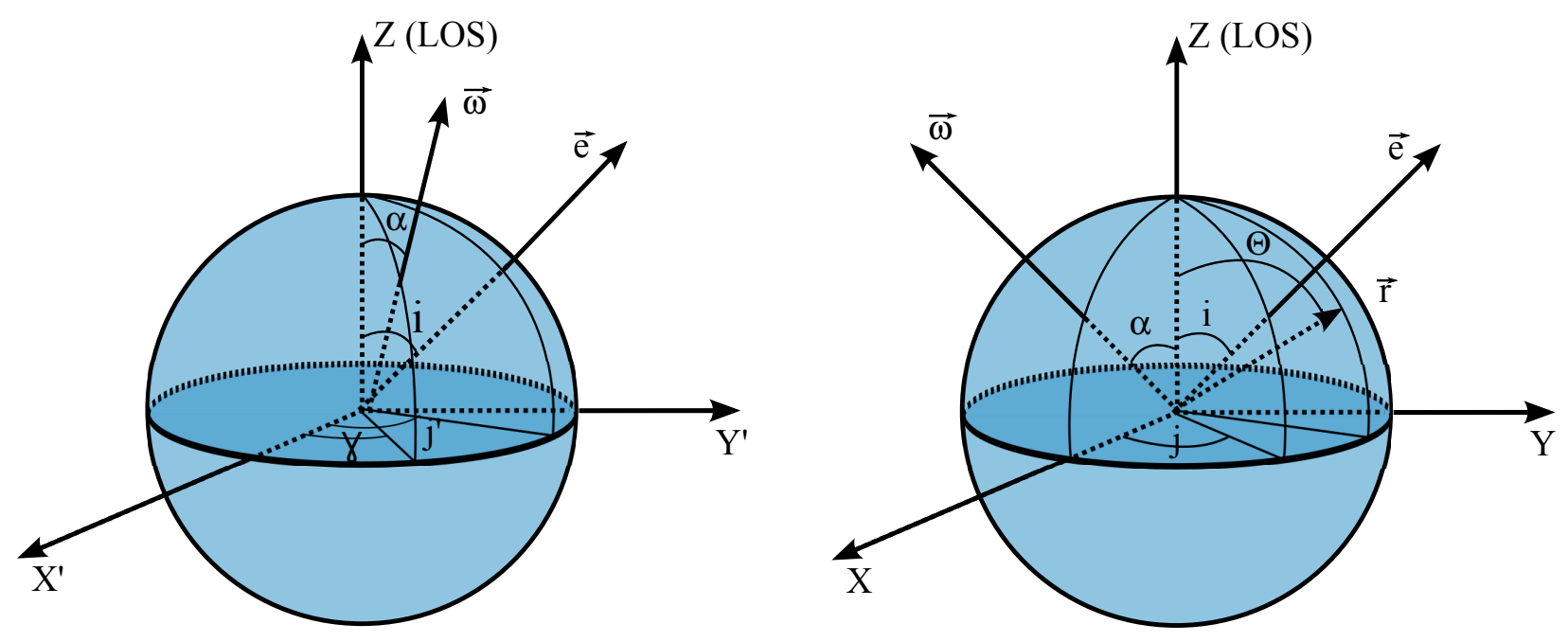

Fig. 2.- This figure shows the two reference systems used in this work. We consider that the observer is along the $Z$ axis, that is shared between both reference systems. The reference system $X^{\prime} Y^{\prime} Z$ is an arbitrary one defined by the observer, where $X^{\prime}$ defines the reference direction for $Q>0$. In order to simplify the computations, we use the $X Y Z$ reference system where the rotation angular velocity vector is in the $X Z$ plane. The transformation between the two reference systems is given by a standard rotation with an angle $\gamma$. The vector e defines the dipolar magnetic field, while $\mathbf{r}$ defines the position on the stellar surface.

magnetic field and carry out the angular and radial integrals on the visible disk. We end 
up with:

$$
\begin{aligned}
X_{1}(i, j, \alpha, \omega, n, a, b) & =(\omega \sin \alpha)^{n}\left\{A_{1}(i, j, \alpha, \omega, n)\left[(1-a-b) F_{1}(n)+a F_{2}(n)+b F_{3}(n)\right]\right. \\
& +B_{1}(i, j, \alpha, \omega, n)\left[(1-a-b) F_{3}(n)+a F_{4}(n)+b F_{5}(n)\right] \\
& \left.+C_{1}(i, j, \alpha, \omega, n)\left[(1-a-b) F_{2}(n+1)+a F_{3}(n+1)+b F_{4}(n+1)\right]\right\} \\
X_{2}(i, j, \alpha, \omega, n, a, b)= & (\omega \sin \alpha)^{n}\left\{A_{2}(i, j, \alpha, \omega, n)\left[(1-a-b) F_{1}(n+2)+a F_{2}(n+2)+b F_{3}(n+2)\right]\right. \\
& +B_{2}(i, j, \alpha, \omega, n)\left[(1-a-b) F_{1}(n+4)+a F_{2}(n+4)+b F_{3}(n+4)\right] \\
& +C_{2}(i, j, \alpha, \omega, n)\left[(1-a-b) F_{2}(n+1)+a F_{3}(n+1)+b F_{4}(n+1)\right] \\
& +D_{2}(i, j, \alpha, \omega, n)\left[(1-a-b) F_{2}(n+3)+a F_{3}(n+3)+b F_{4}(n+3)\right] \\
& \left.+E_{2}(i, j, \alpha, \omega, n)\left[(1-a-b) F_{1}(n)+a F_{2}(n)+b F_{3}(n)\right]\right\} \\
& =(\omega \sin \alpha)^{n}\left\{A_{3}(i, j, \alpha, \omega, n)\left[(1-a-b) F_{1}(n+2)+a F_{2}(n+2)+b F_{3}(n+2)\right]\right. \\
& +B_{3}(i, j, \alpha, \omega, n)\left[(1-a-b) F_{1}(n+4)+a F_{2}(n+4)+b F_{3}(n+4)\right] \\
+ & C_{3}(i, j, \alpha, \omega, n)\left[(1-a-b) F_{2}(n+1)+a F_{3}(n+1)+b F_{4}(n+1)\right] \\
X_{3}(i, j, \alpha, \omega, n, a, b) & D_{3}(i, j, \alpha, \omega, n)\left[(1-a-b) F_{2}(n+3)+a F_{3}(n+3)+b F_{4}(n+3)\right] \\
+ & \left.E_{3}(i, j, \alpha, \omega, n)\left[(1-a-b) F_{1}(n)+a F_{2}(n)+b F_{3}(n)\right]\right\} \\
X_{4}(i, j, \alpha, \omega, n, a, b) & (\omega \sin \alpha)^{n} A_{4}(i, j, \alpha, \omega, n)\left[(1-a-b) F_{1}(n)+a F_{2}(n)+b F_{3}(n)\right] .
\end{aligned}
$$

These functions depend on the orientation of the dipole given by the angles $i$ and $j$, the angular velocity parameters and the parameters of the CLV and the index $n$ of the summation. The following functions take into account the dependence on the orientation of 
the dipole and only depend on angular integrals:

$$
\begin{aligned}
A_{1}(i, j, \alpha, \omega, n) & =S(n) \cos i \\
B_{1}(i, j, \alpha, \omega, n) & =-3 S(n) \cos i \\
C_{1}(i, j, \alpha, \omega, n) & =-3 S(n+1) \sin i \sin j \\
A_{2}(i, j, \alpha, \omega, n) & =3 \cos ^{2} i[3 S(n)-8 S(n+2)]-6 \sin ^{2} i \cos ^{2} j S(n)+6 S(n+2) \\
B_{2}(i, j, \alpha, \omega, n) & =9 \cos ^{2} i[S(n+2)-S(n)+2 S(n+4)] \\
& +9 \sin ^{2} i \cos ^{2} j[S(n)-4 S(n+2)+4 S(n+4)] \\
C_{2}(i, j, \alpha, \omega, n) & =6 \cos ^{2} \sin i \sin j S(n+1) \\
D_{2}(i, j, \alpha, \omega, n) & =18 \cos i \sin i \sin j[S(n+1)-2 S(n+3)] \\
E_{2}(i, j, \alpha, \omega, n) & =\sin ^{2} i \cos (2 j) S(n) \\
A_{3}(i, j, \alpha, \omega, n) & =-3 \sin ^{2} i \cos j \sin j S(n) \\
B_{3}(i, j, \alpha, \omega, n) & =18 \sin ^{2} i \cos j \sin j[S(n+2)-S(n+4)] \\
C_{3}(i, j, \alpha, \omega, n) & =-3 \cos i \sin i \cos j S(n+1) \\
D_{3}(i, j, \alpha, \omega, n) & =18 \cos i \sin i \cos j[S(n+1)-S(n+3)] \\
E_{3}(i, j, \alpha, \omega, n) & =\sin ^{2} i \cos j \sin j S(n) \\
A_{4}(i, j, \alpha, \omega, n) & =S(n) \\
&
\end{aligned}
$$

with the angular integral $S(n)$ given by:

$$
S(n)=\int_{0}^{2 \pi} d \theta \sin ^{n} \theta=\left[1+(-1)^{n}\right] \sqrt{\pi} \Gamma\left(\frac{1+n}{2}\right) \Gamma^{-1}\left(1+\frac{n}{2}\right),
$$

which is valid for $n>-1$. Likewise, the radial integrals $F_{i}(n)$ are independent on the 
orientation of the dipole and are computed from:

$$
\begin{aligned}
& F_{1}(n)=\int_{0}^{1} \rho^{n+1} d \rho=\frac{1}{2+n} \\
& F_{2}(n)=\int_{0}^{1} \sqrt{1-\rho^{2}} \rho^{n+1} d \rho=\frac{\sqrt{\pi}}{4} \Gamma\left(1+\frac{n}{2}\right) \Gamma^{-1}\left(\frac{5+n}{2}\right) \\
& F_{3}(n)=\int_{0}^{1}\left(1-\rho^{2}\right) \rho^{n+1} d \rho=\frac{1}{8+6 n+n^{2}} \\
& F_{4}(n)=\int_{0}^{1}\left(1-\rho^{2}\right) \sqrt{1-\rho^{2}} \rho^{n+1} d \rho=\frac{3 \sqrt{\pi}}{8} \Gamma\left(1+\frac{n}{2}\right) \Gamma^{-1}\left(\frac{7+n}{2}\right) \\
& F_{5}(n)=\int_{0}^{1}\left(1-\rho^{2}\right)^{2} \rho^{n+1} d \rho=\frac{8}{(2+n)(4+n)(6+n)},
\end{aligned}
$$

which are valid if $n>-2$.

Once the expressions for $X_{i}(n)$ have been particularized to the case of a rotating dipole, it is possible to analyze in detail the shape of the profiles according to Eq. (16). We analyze the symmetry properties of the circular polarization profile, although this can be extended easily to the linear polarization profiles. Recall that the profile $\psi(\lambda)$ is a symmetric function of wavelength with respect to the rest wavelength of the line, while $\psi^{\prime}(\lambda)$ is antisymmetric. According to the properties of the Hermite polynomials, the terms with $n=0,2,4, \ldots$ in the expression for $\overline{B_{z} f \mathcal{H}}$ in Eq. (18) are symmetric, while those with $n=1,3,5, \ldots$ are always antisymmetric. Likewise, for $\overline{B_{z} f \mathcal{H}^{\prime}}$, the terms with $n=0,2,4, \ldots$ are antisymmetric, while those with $n=1,3,5, \ldots$ are symmetric. Consequently, the polarized flux $F_{V}(\lambda)$ can be written as the addition of two functions, one symmetric and the other one antisymmetric:

$$
F_{V}(\lambda)=F_{V}^{\text {sym }}(\lambda)+F_{V}^{\text {antisym }}(\lambda),
$$

where

$$
\begin{aligned}
F_{V}^{\mathrm{syy}}(\lambda) & =C \lambda_{0}^{2} g\left[\psi^{\prime}(\lambda){\overline{B_{z} f \mathcal{H}^{\text {antisym }}}}+\psi(\lambda){\overline{B_{z} f \mathcal{H}^{\prime}}}_{\text {sym }}\right] \\
F_{V}^{\text {antisym }}(\lambda) & =C \lambda_{0}^{2} g\left[\psi^{\prime}(\lambda){\overline{B_{z} f \mathcal{H}_{\text {sym }}}}+\psi(\lambda){\overline{B_{z} f \mathcal{H}^{\prime}}}_{\text {antisym }}\right] .
\end{aligned}
$$

Taking into account the definition of the $X_{1}(n)$ function of Eq. (28), it is easy to verify that the symmetric contribution to $F_{V}(\lambda)$ depends only on $\sin i \sin j$, while the antisymmetric 
contribution depends only on $\cos i$. Consequently, we will find standard antisymmetric circular polarization profiles whenever $i=0$, while they will be fully symmetric when $i=90^{\circ}$.

Stellar rotation is taken into account by keeping fixed the LOS and rotating the vector e around the angular rotation velocity $\boldsymbol{\omega}$. This is accomplished by computing the new rotated dipolar moment unit vector as:

$$
\mathbf{e}_{\mathrm{rot}}=\mathbf{R e}
$$

where the rotation matrix is given by:

$$
\mathbf{R}=\left[\begin{array}{ccc}
\cos \phi+\sin ^{2} \alpha(1-\cos \phi) & -\cos \alpha \sin \phi & \sin \alpha \cos \alpha(1-\cos \phi) \\
\cos \alpha \sin \phi & \cos \phi & -\sin \alpha \sin \phi \\
\sin \alpha \cos \alpha(1-\cos \phi) & \sin \alpha \sin \phi & \cos \phi+\cos ^{2} \alpha(1-\cos \phi)
\end{array}\right]
$$

with $\phi$ indicating the stellar rotation phase and taking values between 0 and $2 \pi$. Note that our initial of phases is an arbitrary position of the vectors, while using the standard notation, the initial phase coincides with the $\boldsymbol{\omega}$ and e vectors lying in the same plane parallel to the $Z$ axis. This implies a phase shift between our $\phi$ angle and the standard definition that is given by:

$$
\tan \phi_{0}=-\frac{\sin i \sin j}{\cos \alpha \sin i \cos j-\sin \alpha \cos i} .
$$

Finally, the inclination and azimuth angles with respect to the $X Y Z$ reference system are obtained using the standard relations, taking into account that $\mathbf{e}_{\mathrm{rot}}$ is a unit vector:

$$
i_{\text {rot }}=\arccos \left[\left(e_{\mathrm{rot}}\right)_{z}\right], \quad j_{\mathrm{rot}}=\arctan \frac{\left(e_{\mathrm{rot}}\right)_{y}}{\left(e_{\mathrm{rot}}\right)_{x}}
$$

\section{Technicalities}

All the functions needed in the previous equations are well behaved and can be computed numerically without much complexity. The main difficulty resides on the fact 
that, if the rotation velocity $\omega \sin \alpha$ is large, the convergence of the expansion in Eqs. (18) is slow and oscillating, thus making it hard to compute. The main problem is that the Hermite polynomials grow in amplitude while the coefficients decrease slow. Only after many terms (the number of which depend on the ratio between the rotation velocity and the broadening), the coefficients start to compensate for the increase in the amplitude of the Hermite polynomials and convergence is eventually obtained. This is a well-known defect of the Gram-Charlier expansion (e.g., Blinnikov \& Moessner 1998). When the rotation velocity is much larger than the Doppler width of the local spectral line (larger than a factor $\sim 8$ ), the compensation between the amplitude increase of the Hermite polynomials and the coefficients of the series expansion occurs for very large values of $n$ [see Eqs. (18)]. At this moment, the double precision computations start to become unprecise and the terms in the expansion do not compensate adequately. This purely numerical drawback can be overcome if arbitrary precision methods are used or if more elaborate methods like the Edgeworth expansion are adapted to our problem (Blinnikov \& Moessner 1998). In any case, we have been able to numerically compute expansions up to $\omega \sin \alpha \sim 8 \sigma c / \lambda_{0}$. In other words, our method with a double precision summation of the expansions shown in Eqs. (18) is presently applicable for rotation velocities not larger than 8 times the Doppler width of the local spectral line.

In any case, it is advantageous to use the following set of modified Hermite polynomials:

$$
H e_{n}^{\star}(x)=\left(\frac{\lambda_{0}}{\sigma c}\right)^{n} \frac{1}{n !} H e_{n}(x),
$$

which fulfill the following recursion formula:

$$
H e_{n}^{\star}(x)=\left(\frac{\lambda_{0}}{\sigma c}\right) \frac{1}{n} x H e_{n-1}(x)-\left(\frac{\lambda_{0}}{\sigma c}\right)^{2} \frac{1}{n} H e_{n-2}(x) .
$$

Computing the Hermite polynomials with this recursion formula, the expansions of Eqs. (18) can be extended to much larger values of $n$. The advantage of using the modified 
$H e_{n}^{\star}(x)$ Hermite polynomials is that their amplitude grow much slower with $n$ than $H e_{n}(x)$ due to the inclusion of the term $\lambda_{0} /(\sigma c)$ and $n$ !. This facilitates the computation of each term in the expansions without numerical overflows. We note that the Hermite polynomials are computed using their recursion relations (Abramowitz \& Stegun 1972).

From the computational point of view, once the rotation angular velocity of the star is set, the wavelength dependence of Eqs. (18) and their derivatives in terms of the Hermite polynomials can be precomputed once and used in all subsequent calculations. This greatly accelerates the process because only the $X_{i}(i, j, n)$ functions have to be computed for a given orientation of the dipolar moment and added until convergence.

A free computer program in IDL is available to compute the observed Stokes fluxes for a rotating dipole using the expressions in this papen2. In this non-optimized version of the code, we synthesize profiles evaluated at 500 wavelength points with a step of $8 \mathrm{~m} \AA$ in $\sim 35-40 \mathrm{~ms}$.

\section{Illustrative examples}

Given our assumptions (weak-field, weak-line, fixed center-to-limb variation, global dipolar field), one of the most direct applications of our equations is to study the magnetism of hot stars. For this reason, we use typical values of the broadening and rotation velocity of such stars (e.g., Petit \& Wade 2012). We show in Fig. 3 two examples for a dipolar magnetic field with different field configurations. Both computations share the parameters for the $\operatorname{CLV}(a=0.2$ and $b=0.2)$, the magnetic field strength in the pole $\left(B_{p}=500 \mathrm{G}\right)$ and the rotation velocity on the surface $\left(v=40 \mathrm{~km} \mathrm{~s}^{-1}\right)$. The artificial selected spectral line is centered at $\lambda_{0}=5000 \AA$ and has an effective Landé factor of $g=3$ and its equivalent for

\footnotetext{
${ }^{2}$ http://www.iac.es/project/magnetism/dipole
} 
linear polarization of $G=9$. Since the magnetic field takes its maximum value at the poles, the spectral line can be safely considered in the weak-field regime. The line broadening is

set to $0.1 \AA$, typical of an observation at a spectral resolution of $R=65000$ of a line with a microturbulent width of $\sim 5 \mathrm{~km} \mathrm{~s}^{-1}$. These values of the broadening and rotation velocity are close to the numerical limit that we presently have for computing the summations of Eqs. (18). Given this limitation, our scheme is presently not applicable to fast rotators with narrow local absorption profiles. The computed intensity flux normalized to the continuum flux is shown in the upper panel of each figure. It is independent of the phase since, under the weak field regime, the intensity does not depend on the magnetic field and we have assumed that the star is structureless. However, it takes into account the rotation broadening and the presence of a CLV. Concerning the linear polarization fluxes, we see maximum amplitudes around 0.02\%, while this increases to values around 100 times larger for circular polarization. This is a direct consequence of the fact that linear polarization appears only in second order.

\section{Conclusions}

We have generalized the expressions for the Stokes parameters in the weak-field weak-line regime to the stellar case in which rotation is taken into account. This produces an intensity flux profile that is broadened by rotation while it is insensitive to the magnetic field. As a consequence, our formalism can only be applied to stars in which the intensity profile is not observed to be modulated by rotation. However, the circular and linear polarized fluxes are sensitive to the topology of the magnetic field. We have demonstrated that the inclusion of Doppler shifts generates a dependence of circular polarization of the intensity flux itself, and not only on its wavelength derivative. Likewise, the linear polarization profiles depend now on the first and second wavelength derivative and on the 
intensity flux itself. We have verified also that the expressions developed in this paper simplify to those obtained by Martínez González et al. (2012) in the non-rotating dipole case. The general weak-field expressions have been particularized to a rotating dipole. The extension to a more general multipolar magnetic field is relatively easy and can be done fast if analytical expressions for the angular and radial integrals exist. In subsequent papers of this series we will deal with thin magnetized disks in Keplerian rotation and unresolved structures in the quiet Sun. Likewise, we will also explore the possibility of using more elaborate expansions to improve the convergence.

We thank R. Manso Sainz for fruitful discussions. Financial support by the Spanish Ministry of Economy and Competitiveness through projects AYA2010-18029 (Solar Magnetism and Astrophysical Spectropolarimetry) and Consolider-Ingenio 2010 CSD2009-00038 is gratefully acknowledged. 

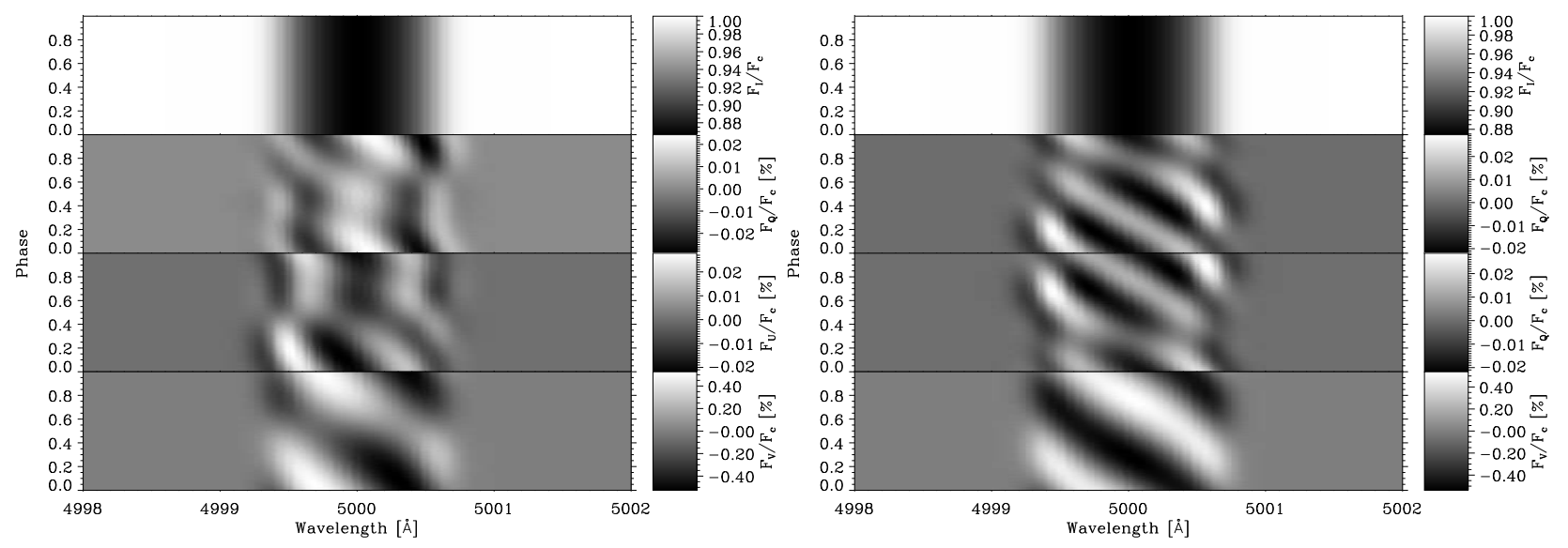

Fig. 3. - Examples of the flux normalized to the continuum intensity flux for a full rotational phase. Both examples share $a=0.2, b=0.2, B_{p}=500 \mathrm{G}$ and $\omega R_{\text {star }}=40 \mathrm{~km} \mathrm{~s}^{-1}$, where $R_{\text {star }}$ is the stellar radius. The left panel corresponds to the case $i=45^{\circ}, j^{\prime}=80^{\circ}, \alpha=70^{\circ}$ and $\gamma=70^{\circ}$. The right panel has $i=45^{\circ}, j^{\prime}=30^{\circ}, \alpha=90^{\circ}$ and $\gamma=20^{\circ}$. All polarized fluxes are given in percentages. 


\section{REFERENCES}

Abramowitz, M., \& Stegun, I. A. 1972, Handbook of Mathematical Functions (New York: Dover)

Aznar Cuadrado, R., Jordan, S., Napiwotzki, R., Schmid, H. M., Solanki, S. K., \& Mathys, G. 2004, A\&A, 423, 1081

Bagnulo, S., Szeifert, T., Wade, G. A., Landstreet, J. D., \& Mathys, G. 2002, A\&A, 389, 191

Blinnikov, S., \& Moessner, R. 1998, A\&A, 130, 193

Claret, A. 2000, A\&A, 363, 1081

Cox, A. N. 2000, Allen's Astrophysical Quantities, 4th ed. (New York: Springer Verlag and AIP Press)

de la Cruz Rodríguez, J., \& Socas-Navarro, H. 2011, A\&A, 527, L8

Donati, J.-F., Semel, M., Carter, B. D., Rees, D. E., \& Collier Cameron, A. 1997, MNRAS, 291,658

Heinzel, P. 1978, Bulletin of the Astronomical Institutes of Czechoslovakia, 29, 159

Jordan, S., Werner, K., \& O’Toole, S. J. 2005, A\&A, 432, 273

Kochukhov, O., Makaganiuk, V., \& Piskunov, N. 2010, A\&A, 524, A5+

Landi Degl'Innocenti, E., \& Landolfi, M. 2004, Polarization in Spectral Lines (Kluwer Academic Publishers)

Landolfi, M., Landi degl'Innocenti, E., Landi degl'Innocenti, M., \& Leroy, J. L. 1993, A\&A, 272,285 
Leone, F., Martínez González, M. J., Corradi, R. L. M., Privitera, G., \& Manso Sainz, R. 2011, ApJL, 731, L33+

Lites, B. W., Kubo, M., Socas-Navarro, H., Berger, T., Frank, Z., Shine, R., Tarbell, T., Title, A., Ichimoto, K., Katsukawa, Y., Tsuneta, S., Suematsu, Y., Shimizu, T., \& Nagata, S. 2008, ApJ, 672, 1237

Martínez González, M. J., Manso Sainz, R., Asensio Ramos, A., \& Belluzzi, L. 2012, MNRAS, 419, 153

Martínez Pillet, V., Del Toro Iniesta, J. C., Álvarez-Herrero, A., Domingo, V., Bonet, J. A., González Fernández, L., López Jiménez, A., Pastor, C., Gasent Blesa, J. L., Mellado, P., Piqueras, J., Aparicio, B., Balaguer, M., Ballesteros, E., Belenguer, T., Bellot Rubio, L. R., Berkefeld, T., Collados, M., Deutsch, W., Feller, A., Girela, F., Grauf, B., Heredero, R. L., Herranz, M., Jerónimo, J. M., Laguna, H., Meller, R., Menéndez, M., Morales, R., Orozco Suárez, D., Ramos, G., Reina, M., Ramos, J. L., Rodríguez, P., Sánchez, A., Uribe-Patarroyo, N., Barthol, P., Gandorfer, A., Knoelker, M., Schmidt, W., Solanki, S. K., \& Vargas Domínguez, S. 2011, Sol. Phys., 268,57

Mihalas, D. 1978, in Stellar Atmospheres, Vol. 455

O’Toole, S. J., Jordan, S., Friedrich, S., \& Heber, U. 2005, A\&A, 437, 227

Petit, V., \& Wade, G. A. 2012, MNRAS, 420, 773

Silvester, J., Neiner, C., Henrichs, H. F., Wade, G. A., Petit, V., Alecian, E., Huat, A., Martayan, C., Power, J., \& Thizy, O. 2009, MNRAS, 398, 1505

Solanki, S. K., Barthol, P., Danilovic, S., Feller, A., Gandorfer, A., Hirzberger, J., Riethmüller, T. L., Schüssler, M., Bonet, J. A., Martínez Pillet, V., del Toro Iniesta, 
J. C., Domingo, V., Palacios, J., Knölker, M., Bello González, N., Berkefeld, T., Franz, M., Schmidt, W., \& Title, A. M. 2010, ApJL, 723, L127

Spirock, T. J., Denker, C., Varsik, J., Shumko, S., Qiu, J., Gallagher, P., Chae, J., Goode, P., \& Wang, H. 2001, AGU Spring Meeting Abstracts, 51

Trujillo Bueno, J. 2011, in Astronomical Society of the Pacific Conference Series, Vol. 437, Solar Polarization 6, ed. J. R. Kuhn, D. M. Harrington, H. Lin, S. V. Berdyugina, J. Trujillo-Bueno, S. L. Keil, \& T. Rimmele, 83

Varsik, J. R. 1995, Sol. Phys., 161, 207

Wade, G. A., Donati, J., Landstreet, J. D., \& Shorlin, S. L. S. 2000, MNRAS, 313, 851 